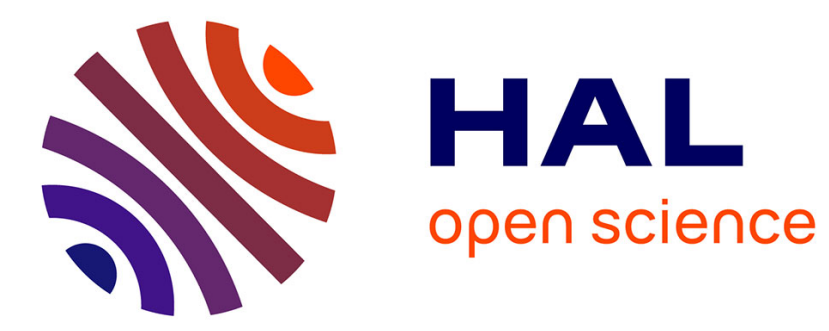

\title{
Anomalous transport due to retroreflection
}

René Lohmann, Beate Horn-Cosfeld, Jakob Schluck, Dominique Mailly, Nima

H Siboni, Hans W Schumacher, Klaus Pierz, Thomas Heinzel, Jürgen Horbach

\section{To cite this version:}

René Lohmann, Beate Horn-Cosfeld, Jakob Schluck, Dominique Mailly, Nima H Siboni, et al.. Anomalous transport due to retroreflection. Physical Review B, 2020, 102, 10.1103/physrevb.102.081302 . hal-03010567

\section{HAL Id: hal-03010567 \\ https://hal.science/hal-03010567}

Submitted on 17 Nov 2020

HAL is a multi-disciplinary open access archive for the deposit and dissemination of scientific research documents, whether they are published or not. The documents may come from teaching and research institutions in France or abroad, or from public or private research centers.
L'archive ouverte pluridisciplinaire HAL, est destinée au dépôt et à la diffusion de documents scientifiques de niveau recherche, publiés ou non, émanant des établissements d'enseignement et de recherche français ou étrangers, des laboratoires publics ou privés. 


\title{
Anomalous transport due to retroreflection
}

\author{
René Lohmann $\odot,{ }^{1}$ Beate Horn-Cosfeld $\odot,{ }^{2}$ Jakob Schluck $\odot,{ }^{2}$ Dominique Mailly, ${ }^{3}$ Nima H. Siboni, ${ }^{4}$ Hans W. Schumacher, ${ }^{5}$ \\ Klaus Pierz $\odot,{ }^{5}$ Thomas Heinzel $\odot,{ }^{2}$ and Jürgen Horbach ${ }^{1}$ \\ ${ }^{1}$ Institut für Theoretische Physik II, Heinrich-Heine-Universität Düsseldorf, Universitätsstraße 1, 40225 Düsseldorf, Germany \\ ${ }^{2}$ Institut für Experimentelle Physik der kondensierten Materie, Heinrich-Heine-Universität Düsseldorf, \\ Universitätsstraße 1, 40225 Düsseldorf, Germany \\ ${ }^{3}$ Université Paris-Saclay, CNRS, Centre de Nanosciences et de Nanotechnologies, 91120 Palaiseau, France \\ ${ }^{4}$ Institut für Theoretische Physik, Technische Universität Berlin, Hardenbergstraße 36, 10623 Berlin, Germany \\ ${ }^{5}$ Physikalisch-Technische Bundesanstalt, Bundesallee 100, 38116 Braunschweig, Germany
}

(Received 13 January 2020; accepted 9 August 2020; published 18 August 2020)

\begin{abstract}
The magnetotransport of an electron gas in a two-dimensional, random arrangement of overlapping retroreflective crosses is studied using a combination of experiment and classical event-driven molecular dynamics simulation. The experimentally measured magnetoconductivity $\sigma_{x x}$ as a function of the magnetic field $B$ displays an anomalous behavior at low $B$ fields, accompanied with an increase of $\sigma_{x x}$ with increasing temperature at $B=0$. The simulations show that at $B=0$ the magnetoconductivity does not exist and is associated with anomalous diffusion in the asymptotic long-time limit that depends on the orientational order of the obstacles. At any finite $B$ field, the motion of the tracer particle is asymptotically diffusive but the diffusion coefficient vanishes with a power law in the limit $B \rightarrow 0$.
\end{abstract}

DOI: 10.1103/PhysRevB.102.081302

Introduction. Anomalous diffusion processes such as suband superdiffusion are ubiquitous in heterogeneous media [1-3]. However, there are only a few particle-based model systems that exhibit anomalous diffusion as an asymptotic process in the long-time limit. A celebrated example in this context is the disordered Lorentz gas (LG) [4] where, in its simplest version, a tracer particle probes the void space of a random arrangement of overlapping spherical objects. In this model, a conductor-insulator transition at a critical obstacle density $n_{c}$ coincides with the percolation transition of the obstacles, implying subdiffusion due to the fractal structure of the free space at $n_{c}$ [3,5-16].

Evidence for a completely different type of asymptotic subdiffusion has been found for Ehrenfest's wind-tree (EWT) model [17]. Here, a point tracer particle moves through the void space of a two-dimensional (2D) random arrangement of overlapping or nonoverlapping squares. The flat boundaries of the squares induce retracing events (or retroreflections) where the tracer particle is reflected back such that even in short-time windows it returns several times to a region where it has been earlier. For overlapping squares, kinetic theory for low densities [18-20] predicts that the multiple backscattering events of the tracer particle result in a meansquared displacement (MSD) with an asymptotic sublinear growth in the long-time limit $\delta r^{2}(t) \propto t^{1-4 n^{\star} / 3}$ (with $n^{\star}=$ $n a^{2}$ a dimensionless obstacle density, where $n$ is the number density of the squares and $2 a$ the length of their diagonals). An early simulation study [21] has qualitatively confirmed the existence of anomalous diffusion in the EWT model with overlapping squares.

These results for the EWT model raise many unsolved questions about anomalous transport in heterogeneous 2D media due to retroreflection. In previous studies, only the lowdensity limit of equally oriented squares has been considered, because this special case can be treated in the framework of kinetic theory. Using a combination of experiment and simulation, we go beyond this regime and consider intermediate and high densities of retroreflective obstacles. Moreover, we study the dependence of retroreflective transport on the obstacle's orientation. We consider 2D systems with random arrangements of overlapping retroreflective crosses (RCs) where effects due to retroreflection are expected to be more pronounced than in the EWT model [22-24].

As we shall see below, a key for the understanding of anomalous subdiffusive transport in retroreflective systems is the introduction of small perturbations from the straight trajectories by an external magnetic field $B$. The $B$ field leads to a circular shape of the tracer particle's trajectory, with the cyclotron radius $R_{\text {cy }} \propto B^{-1}$ acting as a characteristic length scale. We show that even at extremely large cyclotron radii, where the curvature of the trajectories is close to that of the straight lines at $B=0$, the MSD grows always linearly in the long-time limit. As a consequence, we observe a power-law dependence of the magnetoconductivity at extremely low $B$ fields. This anomaly comes along with an asymptotic subdiffusive behavior at $B=0$.

A completely different issue is the investigation of the magnetotransport in an experimental realization of a retroreflective LG in terms of a two-dimensional electron gas (2DEG) with a lithographically imprinted obstacle structure [16,25-35]. In this Rapid Communication, we present experiments on a 2DEG in the classical limit, exposed to a random arrangement of overlapping RCs, and compare them quantitatively to corresponding event-driven molecular dynamics 
(MD) simulations. In the experiments, background disorder prevents the occurrence of pure retroreflection and thus the presence of anomalous transport at $B=0$. However, traces of anomalies due to retroreflection are seen in the behavior of the magnetoconductivity $\sigma_{x x}(B)$, such as an increase of $\sigma_{x x}(B=0)$ with increasing temperature and a characteristic shoulder or peak of $\sigma_{x x}(B)$ at low $B$ fields.

Experiments. A GaAs $/ \mathrm{Al}_{0.3} \mathrm{Ga}_{0.7} \mathrm{As}$ heterostructure with a 2DEG $150 \mathrm{~nm}$ below the surface serves as the raw material. At liquid helium temperatures and after a brief illumination with infrared light, the pristine 2DEG has a density of $n_{e}=2.5 \times 10^{15} \mathrm{~m}^{-2}$ and a typical electron mobility around $340 \mathrm{~m}^{2} / \mathrm{V} \mathrm{s}$, the exact value of which depends on the cooldown cycle as well as on the position of the particular sample on the wafer. This mobility corresponds to a mean free path of $\ell=31 \mu \mathrm{m}$.

A quantum scattering length of $\ell_{q}=530 \mathrm{~nm}$ is extracted from the envelope of the Shubnikov-de Haas oscillations [36], indicating that remote donors are the primary source of scattering. A Hall bar of $100 \mu \mathrm{m}$ width is prepared by optical lithography. Ni/AuGe Ohmic contacts allow electrical access to the 2DEG. Voltage probes of $10 \mu \mathrm{m}$ width, with a spacing of $100 \mu \mathrm{m}$ along the edge of the Hall bar, allow four-terminal measurements.

The Lorentz arrays are patterned by electron beam lithography and subsequent reactive ion etching (etch depth $\approx$ $150 \mathrm{~nm}$ ). They are designed by first generating two sets of random numbers, with pairs of them defining the center coordinates of the obstacles. An RC, composed of two rectangles of size $300 \mathrm{~nm} \times 1850 \mathrm{~nm}$, is attached to each coordinate. Arrays with four number densities $n=0.0625,0.125,0.1875$, and $0.25 \mu \mathrm{m}^{-2}$ are defined by lithography and subsequent reactive argon ion etching with an etch depth of $150 \mathrm{~nm}$, each with orientation order, where all $\mathrm{RC}$ edges form an angle of $\pi / 4$ with respect to the current ( $x$ ) direction, as well as without orientation order, i.e., these angles are randomly distributed. The lateral depletion of the 2DEG at the etched walls extends over a distance which is not known very well. We have estimated a depletion length of $75 \mathrm{~nm}$ [16,37-39], corresponding to RCs of $2 \mu \mathrm{m}$ size and an area of $1.6 \mu \mathrm{m}^{2}$. In Fig. 1, a typical RC and an array thereof is shown. The dimensionless number density $n^{\star}$, defined as the fraction of the area covered by the RCs in the absence of mutual overlaps, takes the values $n^{\star}=0.1,0.2,0.3$, and 0.4 for our arrays.

The transport measurements are carried out in the mixing chamber of a ${ }^{3} \mathrm{He} /{ }^{4} \mathrm{He}$ dilution refrigerator with a base temperature of $25 \mathrm{mK}$ as well as in a ${ }^{4} \mathrm{He}$ gas flow cryostat for temperatures above $1.2 \mathrm{~K}$. Both cryostats are equipped with superconducting solenoids with maximum magnetic fields above $B=8 \mathrm{~T}$. An AC current of amplitude $500 \mathrm{nA}$ and a frequency of $17.7 \mathrm{~Hz}$ is applied, and the voltage drops across the arrays in longitudinal $(x)$ and transverse $(y)$ directions are recorded via the voltage probes using lock-in amplifiers.

Details of the simulation. For the event-driven MD simulations, randomly distributed overlapping RCs are placed in a rectangular box with dimension $200 d_{\mathrm{o}} \times 100 d_{\mathrm{o}}$, assuming periodic boundary conditions (here, the length scale $d_{\mathrm{o}}$ corresponds to $1 \mu \mathrm{m}$ in the experiments). The RCs have the same geometry as those in the experiment (Fig. 1). Both obstacle configurations with equally oriented and randomly
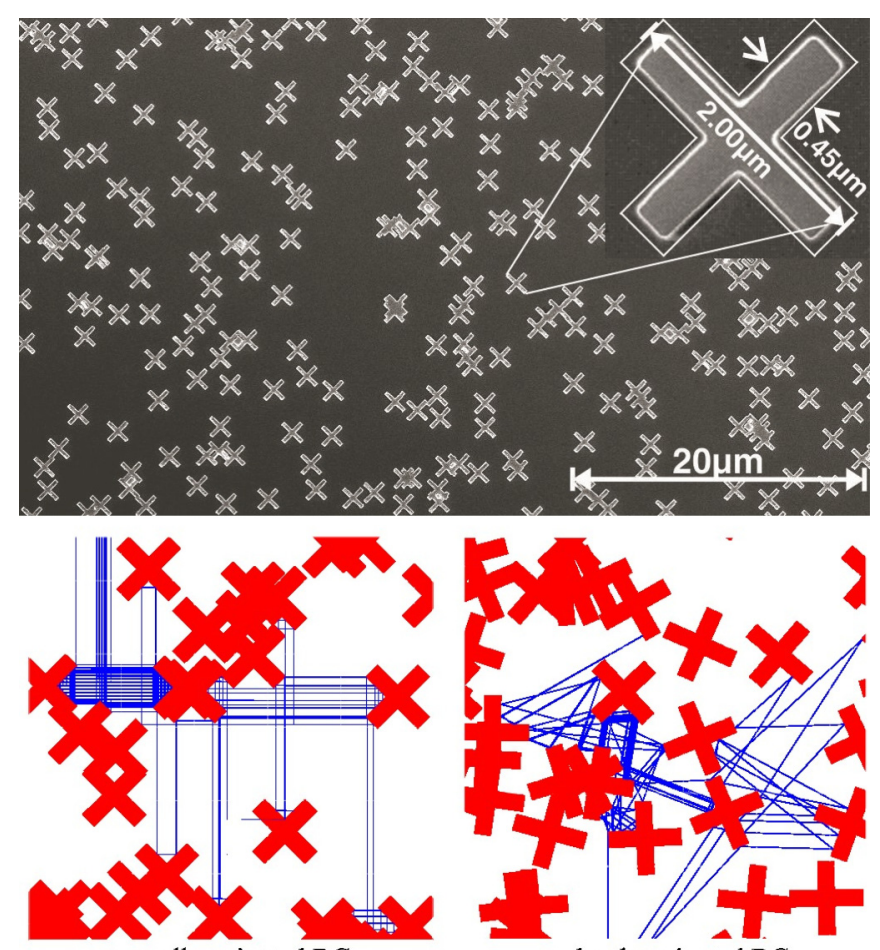

equally-oriented $\mathrm{RCs}$

randomly-oriented $\mathrm{RCs}$

FIG. 1. Upper panel: Scanning electron microscopy image of the experimental system at the density $n^{\star}=0.2$. The inset shows a single $\mathrm{RC}$ and its geometry. Lower panel: Local configurations with equally oriented RCs (left) and randomly oriented RCs (right) at $n^{\star}=0.2$. The blue lines are trajectories, as obtained from the simulation at $B=0$.

oriented RCs are considered. Noninteracting tracer particles (electrons) with a velocity $\vec{v}$ are placed randomly in the free area between the crosses. For each $B$ field and density $n^{\star}$, considered below, we have simulated trajectories for 2000 independent host structures; in each structure we have placed 500 tracer particles. The initial velocity has a random direction and its magnitude is fixed to the Fermi velocity $v_{\mathrm{F}}=|\vec{v}|$. The conversion of the Fermi velocity, the effective electron mass, and the electron charge to the experimental units are given by $v_{F}=2.16 \times 10^{5} \mathrm{~m} / \mathrm{s}, m^{\star}=6.097 \times 10^{-32} \mathrm{~kg}$, and $e=1.6 \times 10^{-19} \mathrm{C}$, respectively (in simulation units, $v_{\mathrm{F}}, m^{\star}$, and $e$ are set to one). In the following, time is given in units of $t_{0}=\frac{d_{0}}{v_{\mathrm{F}}} \approx 4.63 \mathrm{ps}$. A dimensionless magnetic field is introduced via $\tilde{B}=B / B_{0}$, with $B_{0}=m^{\star} v_{\mathrm{F}} /\left(e d_{\mathrm{o}}\right) \approx 0.0823 \mathrm{~T}$.

Results. Figure 1 displays trajectories for equally and randomly oriented RCs at $\tilde{B}=0$ and $n^{\star}=0.2$ from the simulation. In both cases, one can identify retracing paths that, as we shall see below, lead to a subdiffusive transport in the long-time limit at zero magnetic field.

In the simulation, we study the diffusive transport of the tracer particles via the mean-squared displacement (MSD), defined as $\delta r^{2}(t)=\left\langle[\vec{r}(t)-\vec{r}(0)]^{2}\right\rangle$, with $\vec{r}(t)$ the position of the particle at time $t$ and $\langle\cdots\rangle$ an ensemble average. Figure 2(a) shows the MSD at $\tilde{B}=0$ and the different densities for the randomly and equally oriented RCs. The corresponding time dependence of the local exponent $\gamma(t)=$ $d \log \left[\delta r^{2}(t)\right] / d \log (t)$ is displayed in Figs. 2(b) and 2(c). 

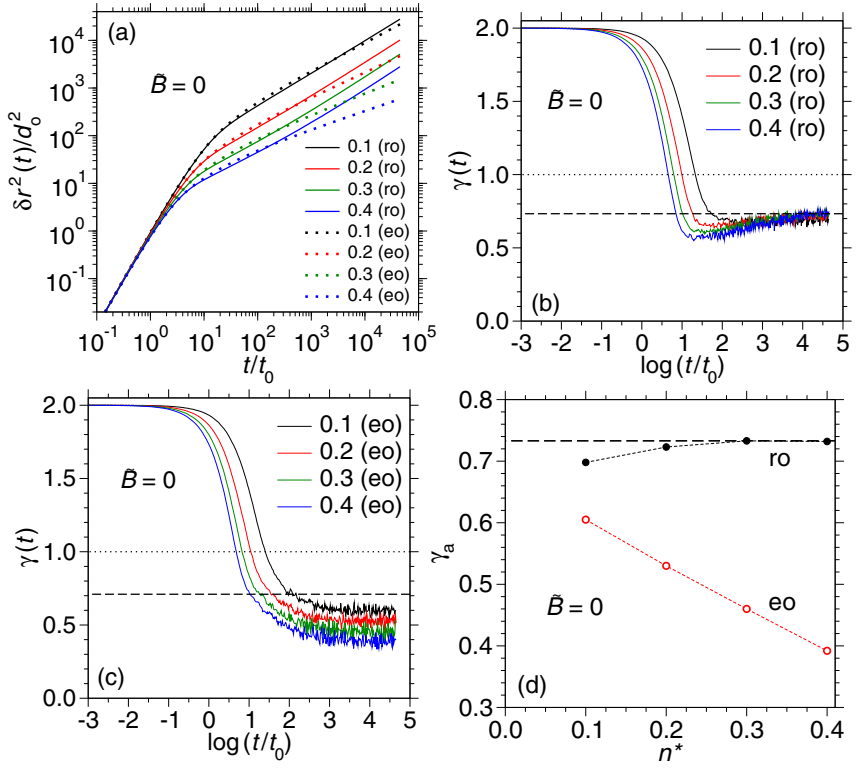

FIG. 2. (a) MSDs, $\delta r^{2}(t)$, at $\tilde{B}=0$ and different densities for randomly oriented RCs ("ro") and equally oriented ones ("eo"), as obtained from the simulation. (b) and (c) show the exponent parameter $\gamma(t)$ corresponding respectively to the randomly oriented and equally oriented RCs. (d) Asymptotic exponent $\gamma_{\mathrm{a}}=\lim _{t \rightarrow \infty} \gamma(t)$ as a function of $n^{\star}$ (see text).

These plots indicate that in all cases the MSDs do not grow linearly in the long-time limit, but as $\delta r^{2}(t) \propto t^{\gamma_{\mathrm{a}}}$ with $\gamma_{\mathrm{a}}<1$. In the case of the randomly oriented RCs, the simulation data suggest a slight increase of $\gamma_{\mathrm{a}}$ towards the constant value $\gamma_{\mathrm{a}} \approx 0.73$ at high density. For the equally oriented RCs, $\gamma_{\mathrm{a}}$ decreases continuously with increasing obstacle density [see Fig. 2(d)].

To compare the behavior at finite magnetic fields to that at $\tilde{B}=0$, we consider the derivative of the MSD (dMSD) $d \delta r^{2}(t) / d t$. In the case of an asymptotic diffusive process, this quantity gives the diffusion coefficient $D=$ $\lim _{t \rightarrow \infty} \frac{1}{4} d \delta r^{2}(t) / d t$. A reduced, dimensionless diffusion coefficient $D^{\star}$ can be obtained from $D=D^{\star} d_{\mathrm{o}} v_{\mathrm{F}}$. This diffusion coefficient is related to the conductivity via $\sigma_{x x}=\frac{n_{e} e^{2} d_{0}}{m^{\star} v_{\mathrm{F}}} D^{\star}=$ $\left(0.486 \times 10^{-2} \Omega^{-1}\right) D^{\star}$.

Figures 3(a) and 3(b) show the dMSD at $n^{\star}=0.2$ for the randomly and equally oriented RCs, respectively (note that we find a similar behavior for the other densities, too). Since for $\tilde{B}=0$ the MSDs exhibit an asymptotic subdiffusive behavior, in this case the dMSD decays as $t^{\gamma_{\mathrm{a}}-1}$ at long times. As we can infer from Figs. 3(a) and 3(b), the dMSDs for sufficiently low $\tilde{B}$ fields first follow exactly the curve for $\tilde{B}=0$ and then, above a timescale $\tau_{B}$ they quickly reach a constant, corresponding to $4 D^{\star}$. For $\tilde{B}$ fields leading to a $\tau_{B}$ smaller than the location of the maximum at $\tau_{\mathrm{m}}$ in the $\mathrm{dMSD}$ for $\tilde{B}=0$, the behavior of the dMSDs changes qualitatively. This happens at about $\tilde{B}_{\mathrm{m}}=0.09$ for $n^{\star}=0.2$. Note that, for $\tilde{B}_{\mathrm{m}}$, the time $\tau_{\mathrm{m}}$ corresponds to the time at which the tracer particle has made on average about 2.3 collisions with RCs. Thus, $\tau_{\mathrm{m}}$ can be considered as the elementary timescale for a retroreflection. The behavior for $\tilde{B}>\tilde{B}_{\mathrm{m}}$ is exemplified in the figures for $\tilde{B}=0.61$. In the latter case, the dMSDs are only in agreement
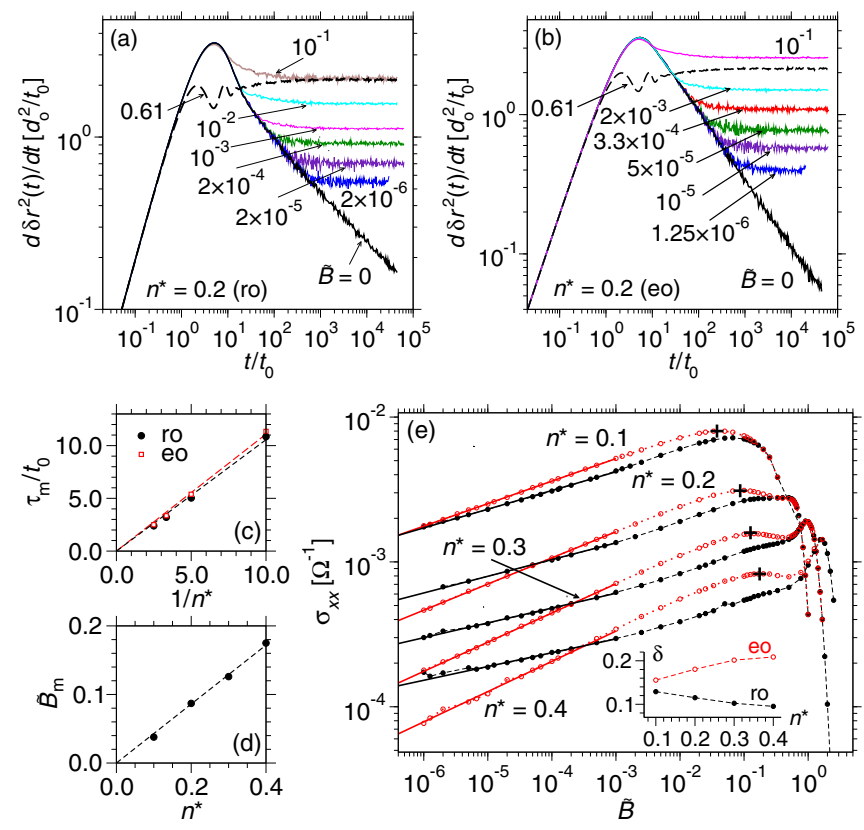

FIG. 3. Derivative of MSD, $d \delta r^{2}(t) / d t$, at $n^{\star}=0.2$ and different magnetic fields, including the case $\widetilde{B}=0$, for (a) the randomly oriented RCs and (b) the equally oriented RCs, as obtained from the simulation. (c) $\tau_{\mathrm{m}}$ as a function of $1 / n^{\star}$. (d) $\tilde{B}_{\mathrm{m}}$ as a function of $n^{\star}$. The dashed lines in (c) and (d) are fits to straight lines. (e) The conductivity $\sigma_{x x}(\tilde{B})$ at the different $n^{\star}$ for randomly oriented RCs (black solid circles) and equally oriented RCs (red open circles). The bold crosses mark the location of $\tilde{B}_{\mathrm{m}}$ at the different $n^{\star}$. The solid lines are fits with power laws $\propto \tilde{B}^{\delta}$. The corresponding exponents $\delta$ as a function of $n^{\star}$ are shown in the inset.

with the dMSD at $\tilde{B}=0$ in the ballistic regime for $t / t_{0}<1$. Around $t / t_{0}=1$, there are some oscillations and the dMSD increases up to a timescale of the order of $10^{3}$, indicating a superlinear increase of the MSD in this time regime.

As Fig. 3(c) indicates, the timescale $\tau_{\mathrm{m}}$ is proportional to the inverse density, $\tau_{\mathrm{m}} \propto 1 / n^{\star}$. The magnetic field $\tilde{B}_{\mathrm{m}}$ is a linear function of $n^{\star}, \tilde{B}_{\mathrm{m}} \propto n^{\star}$ [Fig. 3(d)].

The $\tilde{B}$ dependence of the conductivity $\sigma_{x x}$ is plotted double logarithmically in Fig. 3(e). At small $\tilde{B}$, i.e., for $\tilde{B}<0.01$, a power law, $\sigma_{x x} \propto \tilde{B}^{\delta}$, is seen over about 3-4 orders of magnitude in $\tilde{B}$. While the data for the randomly oriented RCs can be fitted by power laws with a slightly decreasing exponent towards the value $\delta \approx 0.09$ at high densities, for the equally oriented RCs there is an increase of $\delta$ from about 0.15 to 0.21 for the considered densities [inset of Fig. 3(e)], reflecting the behavior of $\gamma_{\mathrm{a}}$ at $\tilde{B}=0$. Around $\tilde{B}_{\mathrm{m}}$ [marked by crosses in Fig. 3(e)], there is a shoulder in the data for the randomly oriented RCs and a local maximum in those for the equally oriented RCs. In this regime, the cyclotron radius is around $1 / \tilde{B}_{\mathrm{m}}$, i.e., of the order of $10 d_{\mathrm{o}}$. Here, the retroreflective character of the trajectories gets lost. Before the monotonic decay towards zero at higher $\tilde{B}$ fields, another maximum can be seen in the curves for $n^{\star} \geqslant 0.2$. This feature is due to the change of the transport from a diffuse scatter by the obstacles to a directed motion along the contour that is also seen in systems with circular obstacles [16]. 

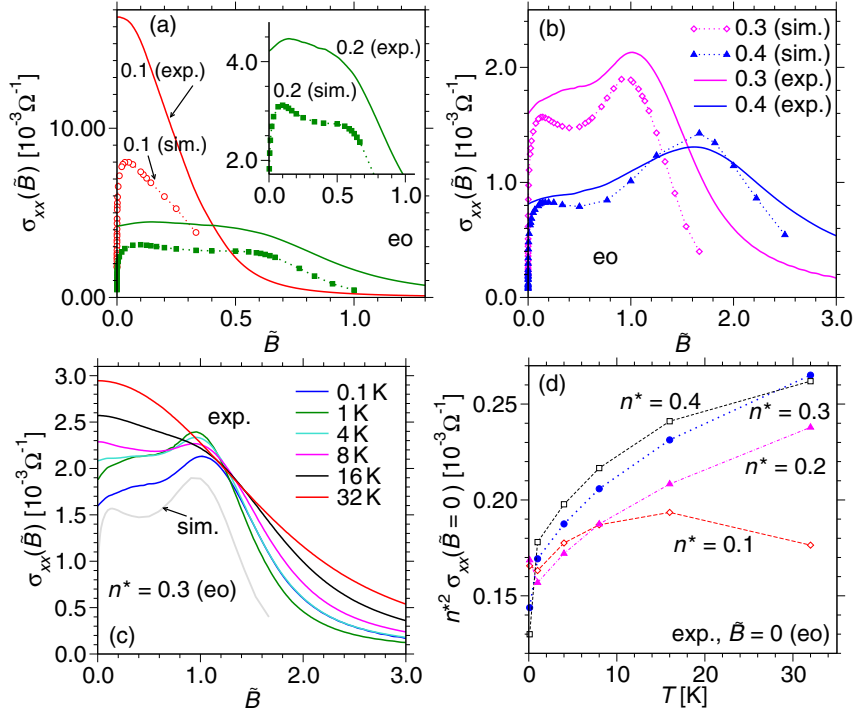

FIG. 4. $\sigma_{x x}(\tilde{B})$, as obtained from experiment and simulation for equally oriented RCs ("eo"), for (a) $n^{\star}=0.1,0.2$ (enlargement in the inset for $n^{\star}=0.2$ ) and (b) $n^{\star}=0.3,0.4$. The experiments are done at the temperature $T=0.1 \mathrm{~K}$. (c) Experimentally measured $\sigma_{x x}(\tilde{B})$ for different $T$ and $n^{\star}=0.3$. (d) Temperature dependence of $n^{\star 2} \sigma_{x x}$ at $\tilde{B}=0$, as obtained from the experiment, for the different densities.

The question now arises as to what extent the transport anomalies, as seen in the simulations, are also visible in the experiment. Figures 4(a) and 4(b) compare the experimentally measured conductivities $\sigma_{x x}(\tilde{B})$ for equally oriented RCs at the different densities and the temperature $T=0.1 \mathrm{~K}$ to corresponding ones from the simulation (for experimental data of randomly oriented RCs, see the Supplemental Material [39]). With increasing RC density, the agreement between simulation and experiment becomes better. At $n^{\star}=0.4$, the discrepancies are always smaller than $15 \%$ for $0.1<\tilde{B}<2.4$ [cf. Fig. 4(b)]. At very low $\tilde{B}$ fields, however, the simulation exhibits the power-law behavior, as discussed above, while the experiment quickly saturates to a finite value in the limit $\tilde{B} \rightarrow$ 0 . The fact that in the experimental system the conductivity at $\tilde{B}=0, \sigma_{x x}(0)$, exists, indicates that there are background scattering processes that perturb the straight trajectories which are seen in the simulation at $\tilde{B}=0$ (cf. Fig. 1). Therefore, the sequence of retracing events that lead to the nonexistence of $\sigma_{x x}(0)$ in the simulation are not present in the experimental system, and thus $\sigma_{x x}(\tilde{B})$ exhibits a regular behavior for $\tilde{B} \rightarrow 0$ and at $\tilde{B}=0$. Note that we can exclude effects due to weak localization in the experimental data, while the onset of Shubnikov-de Haas oscillation is shifted to magnetic fields above $0.2 \mathrm{~T}$ due to the strong scattering at the obstacles [39].

However, the increase of $\sigma_{x x}(\tilde{B})$ towards the shoulder in the interval $0.05 \leqslant \tilde{B} \leqslant 0.2$ [cf. Fig. 3(d)] is a trace of the anomalous transport due to retroreflection in the experimental data at high densities. At $n^{\star}=0.2$ the experimental $\sigma_{x x}(\tilde{B})$ exhibits a maximum at $\tilde{B} \approx 0.1$, in agreement with the simulation [cf. Fig. 4(a)]. With increasing temperature, background scattering events (e.g., due to phonons and local defects in the GaAs substrate) are expected to become more pronounced. In fact, as can be inferred from Fig. 4(c), at $n^{\star}=0.3$, with increasing temperature the shoulder around $\tilde{B}=0.4$ disappears and at $T=32 \mathrm{~K}$ the conductivity decreases monotonically with increasing $\tilde{B}$. While the conductivity maximum caused by the directed motion along the RC's contours [16] is temperature stable up to $16 \mathrm{~K}$, the shoulder around $\tilde{B}=0.4$ is already suppressed at $4 \mathrm{~K}$. This indicates the characteristic length scale of this process to be larger than the one corresponding to the process at $\tilde{B}=1$. Moreover, the conductivity at $\tilde{B}=0$ increases with increasing temperature [cf. Fig. 4(d) where $\sigma_{x x}(T)$ at $\tilde{B}=0$ is multiplied by $n^{\star 2}$ to obtain a better visibility of the differences in the curves at different densities]. At the two highest densities, there is a significant decrease of $\sigma_{x x}$ towards low temperature, reflecting the fact that background scattering processes become less important with decreasing temperature as well as with increasing density. As a consequence, the traces of retroreflective transport become pronounced at low temperatures and high densities.

Discussion and conclusions. We have studied the magnetotransport of a tracer particle (electron) in 2D random arrangements of overlapping RCs, combining event-driven MD simulations with experiments on 2DEG. Our simulations reveal a system with anomalous transport due to retroreflective trajectories of the tracer particle, where at zero magnetic field the conductivity (or diffusion coefficient) does not exist at any density. Instead, subdiffusive transport is seen in the long-time limit. For randomly oriented RCs, the exponent for subdiffusion at $\tilde{B}=0$ slightly increases towards the value $\gamma_{\mathrm{a}} \approx 0.73$ at high density, while for the equally oriented RCs, $\gamma_{\mathrm{a}}$ decreases with increasing density. Our results suggest that a value of $\gamma_{\mathrm{a}}$ well below one is also approached for the equally oriented RCs in the limit of very low densities [cf. Fig. 2(d)]. Thus, one may see a very pronounced anomalous diffusion due to retroreflectivity at very low densities, i.e., in a regime that could be studied in the framework of kinetic theories. This is different in the EWT for equally oriented overlapping squares where the exponent $\gamma_{\mathrm{a}}$ is only slightly different from one in the low-density limit. Thus, systems of disordered arrangements of RCs provide another paradigm for the study of anomalous transport processes via kinetic theory.

Traces of the anomalous transport due to retroreflections can be clearly seen in our experiments. Although background scattering processes cause a perturbation of the retracing events that lead to the nonexistence of the conductivity, several features can be seen in the experimental data that are also present in the classical simulation. Most remarkable is the complex behavior of the $\tilde{B}$ dependence of $\sigma_{x x}$ for $\tilde{B}<2.0$ with two underlying peaks, one being due to retroreflection and the other one due to the change of the transport from a diffusive scatter by the obstacles to a directed motion along their contour. Our study demonstrates that this complex behavior can be fully understood in terms of a classical LG model with RCs.

We thank Dr. E. Neumann (HNF, Forschungszentrum Jülich) for providing scanning electron microscope pictures of the samples, as well as Dr. M. Cerchez and M. Hund for their support of the measurements at sub-Kelvin temperatures. Financial support from Heinrich Heine Universität Düsseldorf and the RENATECH program is gratefully acknowledged. 
[1] R. Metzler and J. Klafter, Phys. Rep. 339, 1 (2000).

[2] M. Sahimi, Flow and Transport in Porous Media and Fractured Rock: From Classical Methods to Modern Approaches (WileyVCH, Weinheim, 2008).

[3] F. Höfling and T. Franosch, Rep. Prog. Phys. 76, 046602 (2013).

[4] H. Lorentz, Proc. K. Ned. Akad. Wet. 7, 438 (1905).

[5] H. van Beijeren, Rev. Mod. Phys. 54, 195 (1982).

[6] A. V. Bobylev, F. A. Maao, A. Hansen, and E. H. Hauge, Phys. Rev. Lett. 75, 197 (1995).

[7] A. V. Bobylev, F. A. Maao, A. Hansen, and E. H. Hauge, J. Stat. Phys. 87, 1205 (1997).

[8] A. V. Bobylev, A. Hansen, J. Piasecki, and E. H. Hauge, J. Stat. Phys. 102, 1133 (2001).

[9] A. Kuzmany and H. Spohn, Phys. Rev. E 57, 5544 (1998).

[10] I. A. Dmitriev, A. D. Mirlin, D. G. Polyakov, and M. A. Zudov, Rev. Mod. Phys. 84, 1709 (2012).

[11] C. P. Dettmann, Commun. Theor. Phys. 62, 521 (2014).

[12] M. Zeitz, K. Wolff, and H. Stark, Eur. Phys. J. E 40, 23 (2017).

[13] W. Schirmacher, B. Fuchs, F. Höfling, and T. Franosch, Phys. Rev. Lett. 115, 240602 (2015).

[14] S. K. Schnyder, M. Spanner, F. Höfling, T. Franosch, and J. Horbach, Soft Matter 11, 701 (2015).

[15] S. K. Schnyder and J. Horbach, Phys. Rev. Lett. 120, 078001 (2018).

[16] N. H. Siboni, J. Schluck, K. Pierz, H. W. Schumacher, D. Kazazis, J. Horbach, and T. Heinzel, Phys. Rev. Lett. 120, 056601 (2018).

[17] P. Ehrenfest, Collected Scientific Papers (North-Holland, Amsterdam, 1959), p. 229.

[18] E. H. Hauge and E. G. D. Cohen, Phys. Lett. A 25, 78 (1967).

[19] E. H. Hauge and E. G. D. Cohen, J. Math. Phys. 10, 397 (1969).

[20] H. Van Beyeren and E. H. Hauge, Phys. Lett. A 39, 397 (1972).

[21] W. W. Wood and F. Lado, J. Comput. Phys. 7, 528 (1971).

[22] S. de Haan, A. Lorke, R. Hennig, M. Suhrke, W. Wegscheider, and M. Bichler, Phys. Rev. B 60, 8845 (1999).

[23] A. Lorke, S. Wimmer, B. Jager, J. P. Kotthaus, W. Wegscheider, and M. Bichler, Physica B 249-251, 312 (1998).
[24] B. G. L. Jager, S. Wimmer, A. Lorke, J. P. Kotthaus, W. Wegscheider, and M. Bichler, Phys. Rev. B 63, 045315 (2001).

[25] E. M. Baskin, L. N. Magarill, and M. V. Entin, Zh. Eksp. Teor. Fiz. 75, 723 (1978) [Sov. Phys. JETP 48, 365 (1978)].

[26] G. M. Gusev, P. Basmaji, Z. D. Kvon, L. V. Litvin, Y. V. Nastaushev, and A. I. Toropov, J. Phys.: Condens. Matter 6, 73 (1994).

[27] G. Nachtwei, G. Lütjering, D. Weiss, Z. H. Liu, K. von Klitzing, and C. T. Foxon, Phys. Rev. B 55, 6731 (1997).

[28] G. Nachtwei, Z. H. Liu, G. Lütjering, R. R. Gerhardts, D. Weiss, K. von Klitzing, and K. Eberl, Phys. Rev. B 57, 9937 (1998).

[29] O. Yevtushenko, G. Lütjering, D. Weiss, and K. Richter, Phys. Rev. Lett. 84, 542 (2000).

[30] A. D. Mirlin, D. G. Polyakov, F. Evers, and P. Wölfle, Phys. Rev. Lett. 87, 126805 (2001).

[31] D. G. Polyakov, F. Evers, A. D. Mirlin, and P. Wölfle, Phys. Rev. B 64, 205306 (2001).

[32] A. Dmitriev, M. Dyakonov, and R. Jullien, Phys. Rev. Lett. 89, 266804 (2002).

[33] V. V. Cheianov, A. P. Dmitriev, and V. Yu. Kachorovskii, Phys. Rev. B 68, 201304(R) (2003).

[34] L. Bockhorn, I. V. Gornyi, D. Schuh, C. Reichl, W. Wegscheider, and R. J. Haug, Phys. Rev. B 90, 165434 (2014).

[35] Y. M. Beltukov and M. I. Dyakonov, Phys. Rev. Lett. 116, 176801 (2016).

[36] T. Ando and Y. Uemura, J. Phys. Soc. Jpn. 36, 959 (1974).

[37] J. Schluck, S. Fasbender, T. Heinzel, K. Pierz, H. W. Schumacher, D. Kazazis, and U. Gennser, Phys. Rev. B 91, 195303 (2015).

[38] J. Schluck, M. Hund, T. Heckenthaler, T. Heinzel, N. H. Siboni, J. Horbach, K. Pierz, H. W. Schumacher, D. Kazazis, U. Gennser, and D. Mailly, Phys. Rev. B 97, 115301 (2018).

[39] See Supplemental Material at http://link.aps.org/supplemental/ 10.1103/PhysRevB.102.081302 for additional experimental data of RC arrays with and without overlaps for two obstacle sizes, and for an estimation of the weak localization correction. 